\title{
NEW OBSERVATIONAL FRONTIERS OF ER UMA (= PG 0943+521) TYPE DWARF NOVAE
}

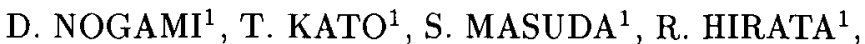 \\ K. MATSUMOTO ${ }^{2}, \mathrm{~K}$. TANABE ${ }^{2}$, T. YOKOO \\ 1. Department of Astronomy, Faculty of Science, \\ Kyoto University, Sakyo-ku, Kyoto 606-01, Japan \\ 2. Astronomical Institute, Osaka Kyoiku University, \\ Asahigaoka, Kashiwara, Osaka 582, Japan
}

The ER UMa-type, including ER UMa, V1159 Ori and RZ LMi, is a subgroup of SU UMa-type dwarf novae. Outbursts of these stars are characterized by: (i) the extremely short recurrence time of the superoutburst ( $\sim 40 \mathrm{~d}$ in ER UMa and V1159 Ori, $\sim 20 \mathrm{~d}$ in RZ LMi), (ii) short outbursts with short recurrence times $(\sim 4 \mathrm{~d})$ between the bright states, and (iii) extremely long duty cycles $(\sim 0.5)$. Assuming that the mass transfer rate from the secondary is ten times higher than that of ordinary SU UMa stars, which was invoked by Kato \& Kunjaya (1995), and a weak tidal torque in the case of RZ LMi, Osaki (1996) showed that the light curves of these stars can be reproduced by the disk instability model, which does not require mass transfer bursts. These indicate that ER UMa, V1159 Ori and RZ LMi are not on the natural extension of SU UMa-type dwarf novae.

The discovery of ER UMa stars has made it natural to interpret that nova-likes showing light variations with photometric periods longer than orbital are in superoutburst all the time. These objects are called 'permanent superhumpers' (PSHs). The mass transfer rates of PSHs can be considered to be even higher than those of ER UMa stars. In the following discussion, we regard ER UMa stars and PSHs as a group of high mass transfer objects.

Let us consider the evolutionary status of these stars. The lifetime of dwarf novae with orbital periods below the period gap is roughly proportional to the inverse of the mass transfer rate from the secondary stars. Thus, the lifetime of ER UMa stars and PSHs is 0.1 times smaller, or less, than that of ordinary SU UMa stars. Since the probability of finding the stars in this stage in CV evolution is proportional to the lifetime, the probability of encountering these stars is estimated as 0.1 of that of ordinary SU UMa stars, or less. However, the observed number ratio of these stars 
(6) to SU UMa stars $(\sim 50)$ has already exceeded that probability and seems to be growing. There are at least two possibilities for the evolutionary status of these stars: (a) these stars are an entirely different population from the ordinary SU UMa stars, and (b) some ordinary SU UMa stars spend some fraction of their life time as ER UMa stars or PSHs.

Suppose that the former possibility, (a), is the case, that is SU UMa stars and a group of ER UMa stars and PSHs have different evolutionary paths and different progenitors. Thus, the number of progenitor ER UMa stars and PSHs may be larger than that of ordinary SU UMa stars. In other words, an important course of the evolution of CVs may have been missed.

In general it is predicted that if the loss of angular momentum is high, that is, if the mass transfer rate is high, then the minimum orbital period should be longer. However, the orbital period of RZ LMi is identical to the minimum value expected from the theory of gravitational-wave radiation. In addition, the orbital periods of ER UMa stars and PSHs seem to be uniformly distributed below the upper edge of the well-known period gap $(\sim 2.8 \mathrm{~h})$. No period gap seems to exist in their orbital period distribution. One possibility to explain this curious distribution is that these stars evolved from pre-CVs to CVs inside the period gap.

In the latter case, (b), even if all of the SU UMa stars experience secular variation of the mass transfer rate as suggested in the hibernation scenario, the fraction of SU UMa stars in the ER UMa star phase or PSH phase to ordinary SU UMa stars is estimated to be 0.2 from the ratio of the observed number of ER UMa stars and PSHs to that of SU UMa stars. To be more realistic, the masses of white dwarfs in SU UMa stars will not always allow SU UMa stars to cause nova explosions. If only some of the SU UMa stars change their mass transfer rates as predicted by the hibernation scenario, the fraction mentioned above becomes still larger, which suggests that the mean mass transfer rate averaged over the lifetime of these systems will far exceed that expected from gravitational-wave radiation. This result is the same as that expected from the former possibility. In either case, these stars are expected to show a different distribution of the orbital period from other SU UMa stars.

The present discussion is based on the data hitherto observed; however it is likely that the data are biased in some way. More precise observational determination of the orbital-period distribution of ER UMa stars and PSHs is highly encouraged, as well as theoretical work on CV evolution taking into account the present discussion.

\section{References}

Kato, T., Kunjaya, C., 1995, PASJ, 47, 163

Osaki, Y., 1996, these proceedings, p127 